\title{
Oral Anticoagulant Utilization in the United States and United Kingdom
}

$\mathrm{J}$ Gen Intern Med 35(8):2505-7 DOI: $10.1007 / \mathrm{s} 11606-020-05904-0$

(c) Society of General Internal Medicine 2020

\section{INTRODUCTION}

Direct oral anticoagulants (DOACs) are used for the prevention and treatment of venous thromboembolism (VTE) and for thromboprophylaxis in adults with atrial fibrillation (AF). ${ }^{1,2}$ For adults with AF, DOACs are the recommended treatment over warfarin. ${ }^{1,2}$ Data from network meta-analyses and observational studies suggest that, compared with other DOACs, apixaban has similar effectiveness and perhaps the lowest risk of major bleeding. ${ }^{1,3}$ This finding has not been confirmed by a randomized clinical trial, and it is unknown how the existing level of evidence has influenced prescribing patterns. The objective of our study was to evaluate trends in oral anticoagulant use in routine care in the United States of America (USA) and United Kingdom (UK).

\section{METHODS}

We analyzed utilization data for warfarin, apixaban, rivaroxaban, dabigatran, and edoxaban using (1) the US Center for Medicare Services (CMS) Part D Prescription Drug Event dataset, ${ }^{4}$ which includes information on outpatient prescriptions dispensed for the approximately $70 \%$ of people enrolled in the Medicare drug benefit plan (2012-2017), and (2) the UK Open Source Database, ${ }^{5}$ which contains prescriptions from nearly all general practices in England (2012-2018). Neither database provides the clinical indication for which the medication was prescribed.

For the US data, we extracted the total number of beneficiaries who received each oral anticoagulant. For the UK data, we extracted the total number of prescriptions dispensed for each anticoagulant. We calculated the change in anticoagulant utilization over time and the proportion of each anticoagulant to total anticoagulant use.

Received September 29, 2019

Revised March 2, 2020

Accepted May 4, 2020

Published online June 8, 2020

\section{RESULTS}

Among US Medicare beneficiaries receiving oral anticoagulants in 2012, 87\% received warfarin; by 2017, this had decreased to $48 \%$. As of 2017, apixaban was the most commonly dispensed DOAC, accounting for $25 \%$ of all oral anticoagulant utilization, followed by rivaroxaban (21\%) (Fig. 1a). The total number of Medicare beneficiaries receiving oral anticoagulants increased by $54 \%$ over the study period.

In the UK, warfarin accounted for $99 \%$ of all oral anticoagulant dispensing in 2012, which had decreased to $47 \%$ by 2018. As of 2018, apixaban was the most frequently dispensed DOAC, accounting for $26 \%$ of all oral anticoagulant utilizations, followed by rivaroxaban (22\%) (Fig. 1b). The total number of oral anticoagulants dispensed increased by $71 \%$ in the UK over the study period.

\section{DISCUSSION}

In this analysis of oral anticoagulant use in the USA and UK, apixaban and rivaroxaban use increased most over time, with apixaban being the most frequently utilized DOAC by the end of the study period. Both far outnumbered utilization of dabigatran or edoxaban, and warfarin dispensing decreased over time.

Apixaban may be the most commonly utilized DOAC because of its favorable risk-benefit profile compared with other DOACs, as reported in observational studies and metaanalyses. ${ }^{1}$ Importantly, no head-to-head randomized trial has directly compared safety and efficacy among DOACs. Neither the US nor European guidelines have indicated a preferred DOAC. ${ }^{2,6}$ Therefore, the similar prescribing pattern between the USA and UK is noteworthy. It suggests that either clinicians find the existing observational data and meta-analyses to be compelling or other factors (such as price, insurance formularies, and patient preference) are driving these similar patterns.

Over the study period, we also observed an increase in total oral anticoagulant utilization in both the USA and UK by $54 \%$ and $71 \%$, respectively. The increase in oral anticoagulant dispensing was likely multifactorial due to (1) an increasing prevalence of AF, (2) growth of the US Medicare and UK populations, (3) increased clinician familiarity and comfort prescribing DOACs, and (4) changes in international guidelines.

This study has several limitations. First, data specifying indication for the oral anticoagulant or length of prescription were not available. Second, these datasets include patients on 
a

Edoxaban Dabigatran $\square$ Rivaroxaban $\square$ Apixaban $\square$ Warfarin

$5 \longrightarrow$

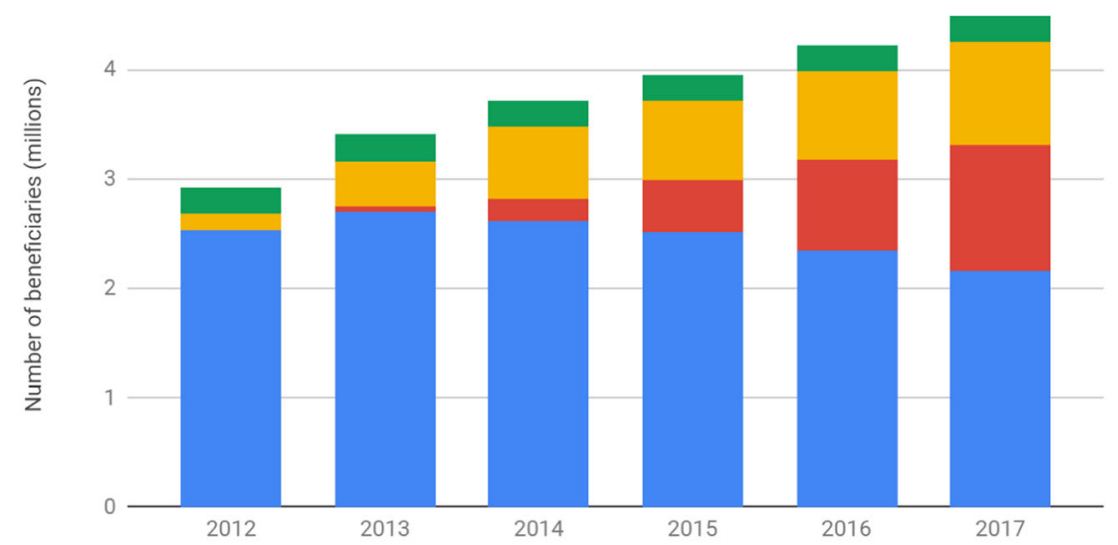

b
Edoxaban
Dabigatran
Rivaroxaban
Apixaban
Warfarin

20

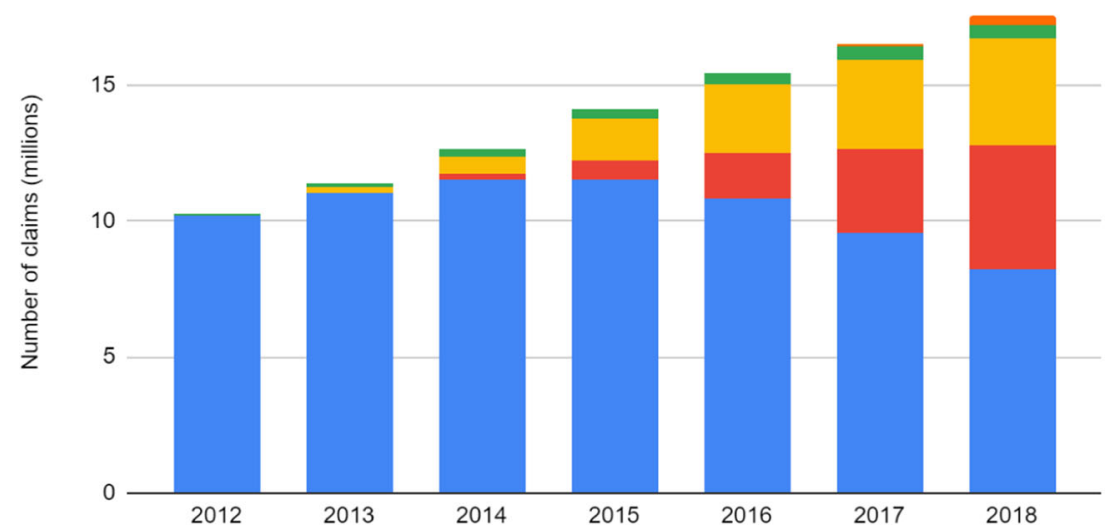

Fig. 1 a Dispensing of each oral anticoagulant between 2012 and 2017 within the US database. There were 0 Medicare beneficiaries who received edoxaban from 2012 to 2014 and fewer than 6000 each year from 2015 to 2017 . b Dispensing of each oral anticoagulant between 2012 and 2018 within the UK database. There were 0 edoxaban prescriptions from 2012 to 2014, fewer than 500 claims in 2015 , and fewer than 13,000 in 2016.

dialysis, for whom warfarin was the preferred oral anticoagulant during the study period. ${ }^{2}$ Third, these datasets do not provide the total number of unique patients included within each database, limiting our ability to discern whether increases in overall prescribing reflect increasing prevalence, changing prescribing patterns, or both. Still, these data offer the clearest picture to date of utilization of oral anticoagulants in the USA and UK following the market entry of DOACs.

Michael Colacci, $M D^{1,2}$

Eric K. Tseng, $M D^{3}$

Chana A. Sacks, $M D, M P H^{4}$

Michael Fralick, $M D, P h D, S M^{1}$

${ }^{1}$ Sinai Health System, Division of General Internal Medicine, Department of Medicine,

Toronto, Canada

${ }^{2}$ Department of Medicine, University of Toronto, Toronto, Ontario, Canada
${ }^{3}$ St. Michael's Hospital, Department of Hematology/ Oncology, University of Toronto,

Toronto, Canada

${ }^{4}$ Division of General Internal Medicine and Mongan Institute, Department of Medicine, Massachusetts General Hospital, Harvard Medical School, Boston, MA, USA

Corresponding Author: Michael Colacci, MD; Department of Medicine, University of Toronto, Toronto, Ontario, Canada (e-mail: michaelfcolacci@gmail.com).

Funding Information Dr. Sacks receives support from the Carney Family Foundation.

Compliance with Ethical Standards:

Conflict of Interest: The authors declare that they do not have a conflict of interest. 


\section{REFERENCES}

1. López-López JA, Sterne JAC, Thom HHZ, et al. Oral anticoagulants for prevention of stroke in atrial fibrillation: systematic review, network metaanalysis, and cost effectiveness analysis. BMJ. 2017;359:j5058.

2. January CT, Wann LS, Calkins H, et al. 2019 AHA/ACC/HRS focused update of the 2014 AHA/ACC/HRS guideline for the management of patients with atrial fibrillation: a report of the American College of Cardiology/American Heart Association Task Force on Clinical Practice Guidelines and the Heart Rhythm Society in collaboration with the Society of Thoracic Surgeons. Circulation. 2019;140(2):e125-e151.

3. Castellucci LA, Cameron C, Le Gal G, et al. Clinical and safety outcomes associated with treatment of acute venous thromboembolism: a systematic review and meta-analysis. JAMA. 2014;312(11):1122-1135.
4. Centers for Medicare \& Medicaid. Medicare provider utilization and payment data: outpatient. Available at: https://www.cms.gov/ResearchStatistics-Data-and-Systems/Statistics-Trends-and-Reports/MedicareProvider-Charge-Data/Outpatient.html. Accessed January 2, 2020.

5. Curtis HJ, Goldacre B. OpenPrescribing: Normalised data and software tool to research trends in English NHS primary care prescribing 19982016. BMJ Open. 2018;8(2):1-10.

6. Kirchhof P, Benussi S, Kotecha D, et al. 2016 ESC guidelines for the management of atrial fibrillation developed in collaboration with EACTS. Rev Esp Cardiol (Engl Ed). 2017;70(1):50.

Publisher's Note: Springer Nature remains neutral with regard to jurisdictional claims in published maps and institutional affiliations. 